important modification-namely, the excision of complete or partial nipple-shaped corneal staphylomata and their substitution by large or complete corneal grafts. Since I described what is perhaps the best method of dealing with corneal staphylomata (Arch. of Ophthal., 1925, Vol. III, No. 2), I have been anxious to develop a better optical procedure in such cases. I feel sure that successful excision of complete corneal staphylomata-selected with regard to the condition of the posterior segment-and their replacement by clear or translucent cornea is only a matter of time. Our ideas on the subject are much more defined and our experience much greater than when the article referred to was written.

While preparing this note for publication, the Amer. Jl. of Ophthal. for October came to hand with an article by Castroviejo which shows that there are various means of accomplishing corneal grafting, but that the best has not yet been evolved. In the same journal the consulting editor discusses professional publicity. This coincidence, even if unintentional, prompts me to say that it is a pity that corneal grafting in England should have received such a vast amount of notice in the daily and popular pictorial lay press in the past year. I gather that the lay press in other parts of the world blindly followed suit. The press in India was no exception and agents seeking news on this subject had to be dealt with rather drastically.

\title{
ANNOTATIONS
}

\section{Blindness in Hereditary Ocular Disease}

In the third of the Dr. Elizabeth Matthai lectures, 1933-34 (Madras University), Lt.-Col. R. E. Wright enumerates a number of hereditary ocular diseases which he claims are preventible if parenthood is abstained from by the affected individuals and by those relatives who, although unaffected themselves, are liable to transmit hereditary disease in some instances.

The publication in these lectures of Wright's observations and ideas on this difficult subject comes at a time when considerable interest is being centred around the means of preventing blindness. The problem of sterilization of the subjects of hereditary ocular diseases has recently been raised by Myles Bickerton in the columns of the Brit. Med. Jl., 1934. It is not surprising that, in a matter of such national and social importance as this, disputes have arisen between persons whose enthusiasm to attempt the absolute elimination of this group of diseases is in conflict with others who, also failing to hold views that savour of scientific balance, pass to the other extreme and complacently 
disregard even the most obvious cases in which some measure of prevention would save the world and the affected individuals from much unnecessary suffering.

The Risley pedigree is quoted by Myles Bickerton in which one man who was blind with aniridia had 13 children similarly affected, 61 out of 63 grandchildren and 39 out of 42 great grandchildren affected. This single individual in the course of 3 generations produced 113 cases of blindness. This pedigree appears on the surface to be incredible, but if thoroughly authenticated it affords an argument in favour of sterilization in such cases. Another example is the high incidence of glioma retinae in some families and affecting several generations. Between these and other extreme cases there is a range of disorders some of which result in blindness, some in seriously impaired vision and in others the vision, although defective, does not interfere with them living a reasonably contented life and enjoying at least some of the things for which eyesight is essential.

Wright quotes the British Medical Journal with reference to the reports of the Prevention of Blindness Committee of the Union of Counties Association for the Blind (Great Britain) issued in 1934 on hereditary blindness, and comments that in these reports the recommendations that persons affected by certain hereditary ophthalmic disorders or in families where these occur or are likely to be transmitted, the words 'abstain from' parenthood are perhaps a little conservative and from his experience of the "criminal carelessness of certain parents" he would feel more inclined to substitute 'be restrained from' parenthood. Wright is quite definite in his belief that birth control measures are impracticable for the uneducated masses in India and therefore has no hesitation in recommending sterilization as the only certain means to prevent propagation of defectives.

This subject abounds with difficulties and even the reports of the genetic experts from the Galton Eugenics Laboratory admit that for technical reasons it cannot be estimated with any degree of accuracy to what extent blindness as a whole could be prevented by limitation of parenthood in individual cases.

Before any dogmatic advice can be given and definite recommendations made in all cases of hereditary ocular disease a great deal of further work must be done particularly on the mode of transmission of these defects. On the elucidation of these facts depends the possibility of giving a fair and reasonably accurate prognosis. There are at present no statistics in ophthalmology which shed any light on the problem of 'penetration,' a term used to denote the frequence with which a normal or pathological character manifests itself in a heterozygous individual. Until these data are forthcoming it would seem that in the present state 
of our knowledge we should be justified in recommending abstention from parenthood only to those individuals who are affected by hereditary diseases which cause serious damage to vision and in whom the mode of transmission is dominant, to their unaffected blood relatives and to those who may transmit the disease in a sex-linked manner, such as the females in families affected by Leber's optic atrophy. In many other hereditary ocular disorders, although it might seem to some a proper course to advise a potential transmitter to forego parenthood, statistics and facts collected to date afford in many instances a groundwork as yet too insecure for a dogmatic stand to be taken.

In this connexion it is apposite to call attention to the masterly report on Hereditary Diseases of the Eye resulting in Blindness, their social consequences and the measures that may be proposed, which was read at the session of the International Association for the Prevention of Blindness on April 5, by Professor Franceschetti of Geneva.

This is too long for abstraction in this place; it should be read in the original. We append Professor Franceschetti's conclusions.

"In conclusion, we shall briefly summarize the measures discussed in detail in the previous chapters, which we believe will enable us to diminish the incidence of hereditary blindness.

a.-Collection of precise and complete statistics,

$b$.- Training of physicians (especially of ophthalmologists) in genetics, and education of authorities and public,

c.-Extension of facilities for pre-marital consultation, and the general introduction of the pre-marital certificate,

d.-Increased use of social workers,

e.-Decrease in consanguineous marriages,

$f$.-Decrease of the transmission of hereditary eye diseases by making sterilization available to the patients.

I am of the opinion that a committee should be named to study the means of realizing these different objectives. In working upon an international plane, this committee should be able to bring together the material which will permit judgment of the efficacy of the steps taken to decrease hereditary blindness in the various countries, and concrete recommendations to be made in the near future."

\section{Research Work in Ophthalmology}

In 1928, Vol. XII, p. 214, we called the attention of our readers to the fact that the management of the Brit. Jl. of Ophthal. was prepared to assist research work in ophthalmology by granting 